\title{
DNA Methylation in the Developing Hippocampus and Amygdala of Anxiety-Prone versus Risk-Taking Rats
}

\author{
Rebecca K. Simmons ${ }^{a}$ Jasmine L. Howard ${ }^{a}$ Danielle N. Simpson ${ }^{a}$ Huda Akil $^{b}$ \\ Sarah M. Clinton ${ }^{\mathrm{a}}$ \\ a Department of Psychiatry and Behavioral Neurobiology, University of Alabama at Birmingham, Birmingham, Ala., \\ and ${ }^{b}$ Molecular and Behavioral Neuroscience Institute, University of Michigan, Ann Arbor, Mich., USA
}

\section{Key Words}

Bred High Responder • Bred Low Responder • DNA methylation - Anxiety · Depression - Hippocampus • Amygdala

\begin{abstract}
All organisms exhibit a wide range of emotional behaviors and interact with the environment in different ways. Some individuals may be more quiet and shy whereas others are more outgoing and adventurous. These temperamental and personality differences can predispose individuals to certain psychopathologies which may be influenced by genetic vulnerability and/or early life experiences. Rodent models can be used to recapitulate emotional reactivity differences, and these models can, in turn, be used to examine potential neurobiological underpinnings of these traits. The present study utilizes two strains of rats that were selectively bred for differences in novelty seeking. High Novelty-Responding (bHR) rats are very active in response to novelty, exhibit exaggerated risk-taking, aggression, impulsivity, and show increased behavioral response to cocaine. Low Novelty-Responding (bLR) rats show increased anxiety, depressive behavior and vulnerability to chronic stress. One way in which the bHR versus bLR behavioral phenotypes may differ is through epigenetic modification of DNA. DNA can be modified through
\end{abstract}

processes such as acetylation or methylation to either enhance or subdue gene expression. This study examines putative differences in methylation levels in the hippocampus and amygdala of developing bHR-bLR rats. Previous research observed widespread gene expression differences in the bLR developing hippocampus, and the current study aims to begin to examine potential epigenetic factors that may contribute to those gene differences. The amygdala was chosen because it is involved in emotional processes, in part through its connections with the hippocampus. Therefore, the present study used in situ hybridization to assess the expression of DNA methyltransferase-1 (DNMT1) mRNA in the hippocampus, amygdala and several other brain areas of bHR and bLR pups at three developmental time points: postnatal days $(P) 7,14$, and 21 . We focused on the first 3 postnatal weeks, in part to parallel our early microarray gene expression work, and because this represents a critical period of brain development, which shapes individuals' lifelong emotional and stress reactivity. We found significant differences in dentate gyrus and $C A 3$ regions of the hippocampus at $P 7$ with no differences seen at P14 or P21. Interestingly, we also found significant bHR-bLR DNMT1 differences at P7 within the lateral, basolateral and medial nuclei of the amygdala, with no difference at P14 and P21, suggesting that the first postnatal week is a critical period for DNA methylation during brain development.

Copyright $\odot 2012$ S. Karger AG, Base

\section{KARGER}

Fax +4161306 1234

E-Mail karger@karger.ch

www.karger.com (c) 2012 S. Karger AG, Basel

0378-5866/12/0341-0058\$38.00/0

Accessible online at:

www.karger.com/dne
Sarah M. Clinton

17207 th Avenue South

SC 745

Birmingham, AL 35233 (USA)

Tel. +1 205975 0312, E-Mail clintons@ @uab.edu 


\section{Introduction}

DNA methylation is the covalent addition of a methyl group to the $5^{\prime}$ position of cytosine residues within $\mathrm{CpG}$ dinucleotides. DNA methylation has traditionally been thought as a mechanism to reduce gene expression although more recent research suggests that methylation can lead to both increased and decreased transcription rates $[1,2]$. This process is dependent upon the presence of methyl donors and methyltransferases which mediate maintenance or de novo DNA methylation. DNA methyltransferases (DNMTs) function by catalyzing the transfer of the methyl group to DNA. Three active methyltransferases have been identified in mammals: DNMT1, DNMT3a, and DNMT3b. DNMT1 has been implicated in maintenance of the methylation patterns; it is involved in copying the preexisting methylation patterns onto new strands of DNA. DNMT 3a/b are both involved in de novo methylation, which establishes new methylation patterns at specific sites within the genome and plays an important role during development. Together these enzymes are critical for regulating gene transcription $[3,4]$.

In recent years, we developed selectively bred lines of Sprague-Dawley rats based on differences in emotional reactivity and exploratory behavior. Rats selected for high novelty exploration (bred High Responders; bHRs) also exhibit exaggerated risk-taking, aggression, impulsivity and proclivity to psychostimulant abuse [5-7] compared to bred Low Responder (bLR) rats, which exhibit enhanced anxiety, depression and vulnerability to chronic stress [8-10]. Overall, the bLR and bHR phenotypes appear to reflect fundamental differences in how they interact with the environment at both the affective and cognitive levels. bHRs exhibit a 'behavioral disinhibition', extensively exploring and interacting with their environment whereas bLRs exhibit 'behavioral over-inhibition', acting highly passive when facing novel or stressful situations. After several rounds of breeding, $>99 \%$ of bHR animals come from bHR parents, and 99\% of bLR animals derive from bLR parents [8], which has granted us the ability to accurately predict the bHR/bLR phenotype during early development before behavioral testing is possible and before experience leads to further differences in neural structure and function. We recently conducted a study where we examined global patterns of gene expression in select brain areas of developing bHR versus bLR rats. Our results revealed dramatic gene expression differences in the developing hippocampus of bHR versus bLR during the first 2 weeks of life [11].

DNMT1 mRNA Expression in

Developing Hippocampus and Amygdala
Given the widespread gene expression differences that we observed in the bHR versus bLR developing hippocampus, the current study aims to begin to examine potential epigenetic factors that may contribute to those gene expression differences. Therefore, the present experiment used in situ hybridization to assess the expression of DNMT1 mRNA in the hippocampus, amygdala, and several other brain areas of bHR and bLR pups at three developmental time points: postnatal days (P) 7, 14 and 21 . We focused on the first 3 postnatal weeks, in part, to parallel our early microarray gene expression work, and because this represents a critical period of brain development, which shapes individuals lifelong emotional and stress reactivity. We focused on the hippocampus since our previous study heavily implicates differences in the establishment of hippocampal circuits contributing to the emergence of the bHR/bLR phenotypes [11] although we also included the amygdala, given its involvement in emotional processes, and considering its substantial interconnectivity with the hippocampus.

\section{Materials and Methods}

\section{Animals}

Animals were acquired from the in-house colony at the University of Michigan where the bLR-bHR lines have been maintained for several years. We previously published a description of our breeding strategy and initial behavioral characterization of the bHR/bLR lines, and have continued to examine the many facets of the bHR-bLR behavioral phenotypes [5-10]. Our original founding population was composed of 60 male and 60 female Sprague-Dawley rats purchased from three separate Charles River Laboratory breeding colonies. Animals were screened for locomotor response to novelty, and males and females within the highest and lowest $20 \%$ of scores from locomotion testing were bred together to generate the first-generation bHR and bLR lines, respectively. For each selected line, 12 litters were maintained at each generation. Adult males and females from each generation were screened for locomotor response to novelty, and the most extreme bHR and bLR animals from within each family were selected to perpetuate the colony. For the present study, male rats from the 6th generation of the in-house colony at the University of Michigan were used. For more details, please see Stead et al. [8]. Rats were kept under a 12:12 light-dark cycle with food and water available ad libitum. Experiments were conducted in accordance with the principles and procedures outlined in the $\mathrm{Na}$ tional Institutes of Health Guidelines for the Care And Use of Animals.

\section{Tissue Collection and in situ Hybridization for DNMT1} $m R N A$

bHR and bLR male pups were sacrificed by rapid decapitation at three developmental time points (P7, P14, and P21). Brains were removed, snap frozen, and stored at $-80^{\circ} \mathrm{C}$. They were cryostat 
sectioned at $12 \mu \mathrm{m}$, immediately thaw-mounted onto Fisherbrand Superfrost Plus Microscope Slides (Fisher Scientific, http:// www.fishersci.com/) and subsequently prepared for in situ hybridization. The expression of DNMT1 mRNA was assessed at $240-\mu \mathrm{m}$ intervals throughout the hippocampus, amygdala (in P7, P14 and P21 brains), and several forebrain regions (at P7 only).

Our in situ hybridization protocol has been previously described [5-7]. Briefly, sections were fixed in $4 \%$ paraformaldehyde at room temperature for $1 \mathrm{~h}$. The slides were then washed $5 \mathrm{~min}$ $\times 3$ in $2 \times$ SSC $(300 \mathrm{~mm} \mathrm{NaCl} / 30 \mathrm{~mm}$ sodium citrate, $\mathrm{pH} 7.2)$. Next, the slides were placed in a solution containing acetic anhydride $(0.25 \%)$ in triethanolamine $(0.1 \mathrm{M}), \mathrm{pH} 8.0$, for $10 \mathrm{~min}$ at room temperature, rinsed in distilled water, and dehydrated through graded ethanol washes (50, 75, 85, 95 and 100\%). After air drying, adjacent tissue sections were hybridized overnight at $55^{\circ} \mathrm{C}$ with a ${ }^{35} \mathrm{~S}$-labeled cRNA probe for DNMT1. Following hybridization, coverslips were removed and the slides were washed with $2 \times$ SSC and incubated for $1 \mathrm{~h}$ in RNaseA at $37^{\circ} \mathrm{C}$, followed by multiple washes in increasingly stringent SSC solutions. Slides were then rinsed in distilled $\mathrm{H}_{2} \mathrm{O}$, dehydrated through graded ethanol washes, air dried, and apposed to Kodak XAR film (Eastman Kodak, Rochester, N.Y., USA). Slides were exposed to film for 7 days.

The following cRNA riboprobe was used for DNMT1 (NCBI reference sequence - D64060, pos. -183-673). Specificity of labeling was confirmed by the absence of signal following hybridization, with sense riboprobes generated for the same positions of the target mRNAs (data not shown). After the 7-day exposure period, autoradiograms were developed and digitized using a ScanMaker 1000XL Pro flatbed scanner (Microtek, Carson, Calif., USA) with LaserSoft Imaging software (AG, Kiel, Germany) at 1,600 dpi. Digitized images were analyzed using ImageJ Analysis Software for PC from NIH. Specific signal, defined as $3.5 \times$ the standard deviation of individual pixel signal values above mean background signal, was converted to optical density and multiplied by the area of signal to produce integrated optical density (IOD) as noted below. The units of IOD are arbitrary units. As long as slides have been processed in parallel and exposed to film for an equivalent period of time, it allows one to effectively compare across experimental groups and/or across brain regions.

\section{In situ Hybridization Data Analysis}

In an effort to standardize measurements of in situ hybridization signal and optical density, we utilized a template for each brain region or subnucleus based on the shape and size of the region. Using these templates, optical density measurements were collected for each brain region (the hippocampal subfields - CA13 and dentate gyrus, and several nuclei of the amygdala: the lateral, basolateral, basomedial, central, medial nuclei as well as the cortical amygdaloid area and amygdalar-hippocampal transition area). These measurements were made in $8-10$ sections spaced $240 \mu \mathrm{m}$ apart across the rostrocaudal extent of the hippocampus and amygdala from the left and right sides of the brain (where possible) and/or from rostrocaudal sections spaced $240 \mu \mathrm{m}$ apart. Optical density values were corrected for background, multiplied by the area sampled to produce the IOD measurement, which was then averaged to produce a single data point for each brain region per animal. These data points were averaged per group and compared statistically.

\section{Statistical Analysis}

Data from the hippocampus, amygdala, and other brain regions were analyzed using ANOVA through SPSS 19.0. In our first analysis, we compared DNMT1 mRNA expression across different areas of the hippocampus or amygdala at the three different developmental time points: P7, P14, and P21. Therefore, we used a two-way ANOVA with hippocampal subregion (or amygdalar nuclei) as one factor, and developmental age as the second factor (bHR/bLR phenotype was not considered in this initial analysis, so data were combined in this initial examination). The second analysis examined the impact of bHR and bLR phenotype on DNMT1 mRNA expression in the hippocampus and amygdala. Here, each brain region (hippocampal subfield, amygdalar nuclei) at each developmental time point was considered separately and we used a oneway ANOVA with bHR/bLR phenotype as the independent factor. In a follow-up study, we compared DNMT1 transcript expression in the caudate putamen, cingulate cortex, septum, and nucleus accumbens of bHR/bLR rats only at P7; here, we used a one-way ANOVA with bHR/bLR phenotype as independent factor. Data were analyzed using SPSS 19.0 for Windows. For all tests $\alpha=0.05$.

\section{Results}

\section{DNMT1 mRNA Expression in the Developing}

Hippocampus and Amygdala

We first sought to ascertain the developmental pattern of DNMT1 mRNA expression in the hippocampus and amygdala at three developmental time points: P7, P14, and P21 (fig. 1). Visual inspection of the autoradiographic films exposed to the in situ hybridization-processed slides clearly reveals a decrement of DNMT1 mRNA levels over time, with higher levels apparent at P7 compared to P14 and P21 in both the hippocampus (top row, fig. 1) and amygdala (bottom row, fig. 1). DNMT1 mRNA expression was quantified in 5 subregions of the hippocampus - CA1, CA2, CA3, upper blade of the dentate gyrus and lower blade of the dentate gyrus (fig. 2a). The two-way ANOVA showed a main effect of subregion $\left(\mathrm{F}_{4,249}=101.252\right.$, $\left.\mathrm{p}>0.001\right)$ and developmental age $\left(\mathrm{F}_{2,249}=29.984, \mathrm{p}>0.001\right)$, and no subregion $X$ age interaction on $\mathrm{IOD}$ measurements in the hippocampus. DNMT1 transcript expression was also quantified at P7, P14, and P21 in 7 nuclei of the amygdala: basolateral, central, cortical amygdaloid, lateral, medial, amygdalar-hippocampal transition area, and basolateral. Here, there was a main effect of age $\left(\mathrm{F}_{2,242}=49.631, \mathrm{p}>\right.$ $0.001)$, effect of nucleus $\left(\mathrm{F}_{6,242}=32.016, \mathrm{p}>0.001\right)$, and age $\times$ nucleus interaction $\left(\mathrm{F}_{12,242}=3.076, \mathrm{p}>0.001\right)$.

\section{DNMT1 mRNA Expression in Developing bHR versus bLR Hippocampus}

DNMT1 mRNA expression was compared between bHR and bLR pups within multiple hippocampal sub- 


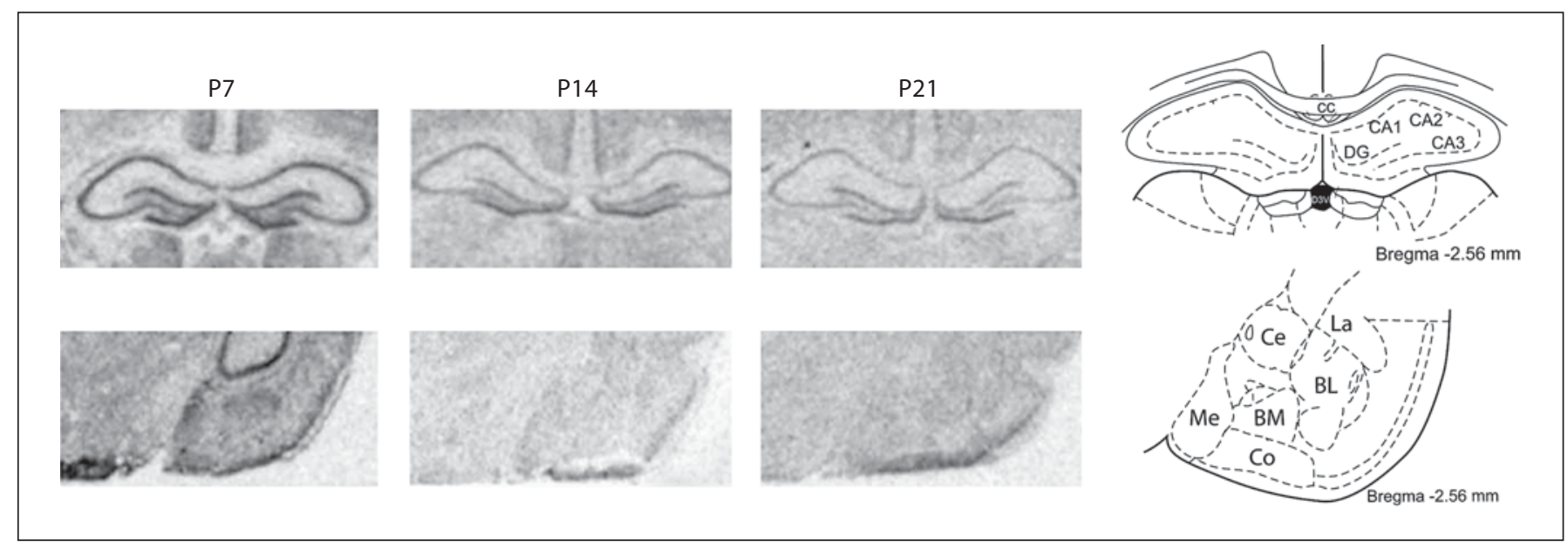

Fig. 1. DNMT1 mRNA expression in hippocampus and amygdala. The first three columns show autoradiograms from representative X-ray films exposed for 7 days after in situ hybridization with an antisense riboprobe against rat DNMT1 mRNA. Images show examples of the hippocampus (top row) and amygdala (bottom row) at P7, P14 and P21. The diagrams in the far right column (adapted from [35]), indicate the subfields of the hippocampus (CA1-3 and dentate gyrus) and several amygdalar nuclei that were examined. $\mathrm{DG}=$ Dentate gyrus; $\mathrm{Ce}=$ central nucleus; $\mathrm{La}=$ lateral nucleus; $\mathrm{BL}=$ basolateral nucleus; $\mathrm{BM}=$ basomedial nucleus; $\mathrm{Me}$ $=$ medial nucleus; $\mathrm{Co}=$ cortical amygdaloid area .

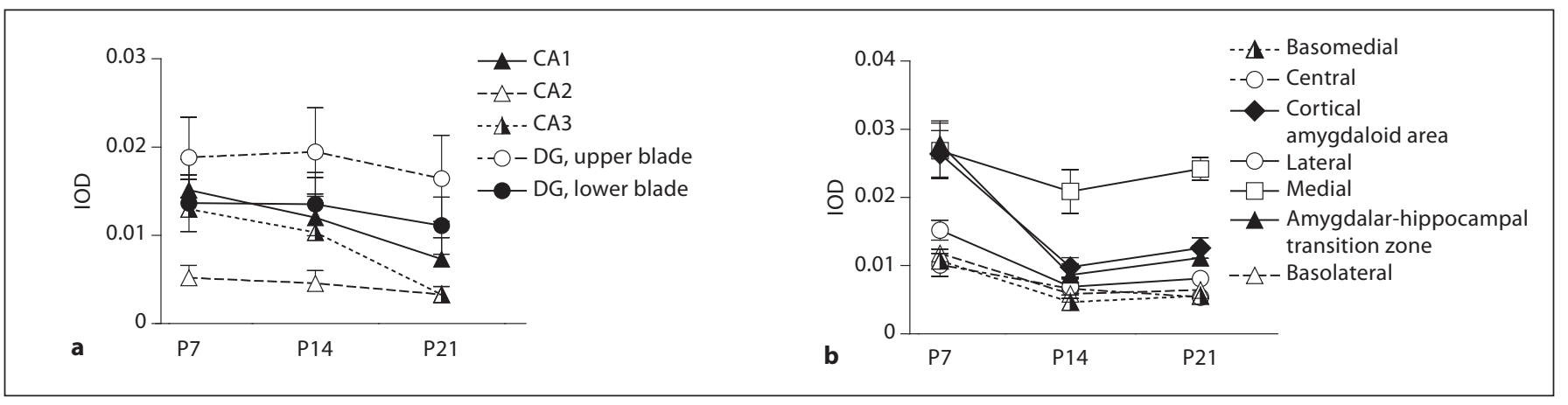

Fig. 2. Developmental pattern of DNMT1 mRNA expression in hippocampus (a) and amygdala (b). DNMT1 mRNA levels were measured in 5 subregions of the hippocampus - CA1-3 and separately in the upper and lower blade of the dentate gyrus (DG), due to functional differences within these regions. Statistical analysis revealed significant differences in DNMT1 expression between the different subfields, and did show a significant effect of developmental age (a). DNMT1 transcript expression was also assessed in the developing amygdala, focusing on 7 nuclei: the basomedial nucleus, central nucleus, cortical amygdaloid area, lateral nucleus, medial nucleus, amygdalar-hippocampal transition zone and basolateral nucleus. Here there was a significant effect of developmental age, with higher DNMT1 levels expressed at P7 relative to P14 and P21 (b). Optical density values were corrected for background, multiplied by the area sampled to produce the IOD measurement, which was then averaged to produce a single data point for each brain region per animal. fields at three developmental time points: P7, P14, and P21. bHR/bLR differences in DNMT1 mRNA expression were discovered, but only within select regions of the hippocampus, and only at P7 (fig. 3a). At P7, bHR pups had increased DNMT1 levels compared to bLRs in the upper blade of the dentate gyrus $\left(\mathrm{F}_{1,83}=8.78, \mathrm{p}>0.01\right)$, but there were no differences at P14 or P21 (fig. 3a), and no DNMT1 expression differences in the lower blade of the dentate gyrus at any of the time points (fig. 3b). bHRs also exhibited higher DNMT1 mRNA levels compared to bLRs within CA3 at P7 $\left(\mathrm{F}_{1,83}=5.845, \mathrm{p}>0.05\right)$, with no bHR/ bLR differences occurring at P14 or P21 (fig. 3d). Con- 


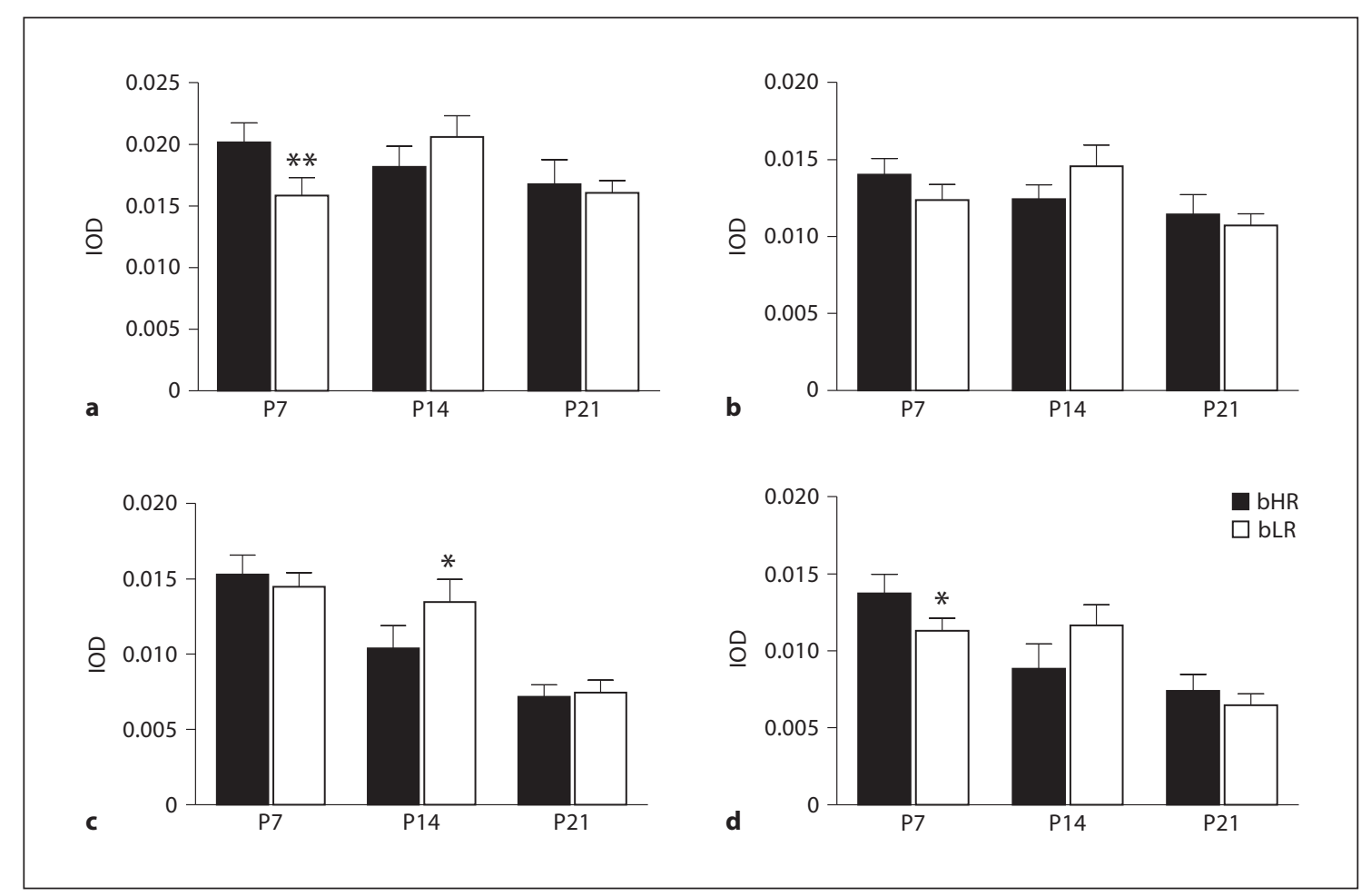

Fig. 3. DNMT mRNA expression in developing bHR versus bLR hippocampus. At P7, bHR pups expressed higher DNMT1 mRNA in the upper dentate gyrus compared to bLR pups. This bHR/bLR difference in the dentate gyrus was restricted to the upper blade (a) as there were no group differences in DNMT1 expression the lower blade (b). At P7, bHRs also showed higher DNMT1 expression compared to bLRs in CA3 (d). While there were no bHR/bLR differences in DNMT1 expression at P7, at P14, bLRs had higher levels compared to bHR (c). There were no group differences at any time point for CA2 (data not shown). ${ }^{*} \mathrm{p}<0.05$, and ${ }^{* *} \mathrm{p}<0.01$.

versely, in the CA1 subregions, bLRs had higher DNMT1 expression compared to bHRs $\left(\mathrm{F}_{1,62}=5.892, \mathrm{p}>0.05\right)$ although this effect was only present at P14 (fig. 3c). No bHR/bLR differences were detected at any time point in the CA2 region (data not shown).

\section{DNMT1 mRNA Expression in Developing bHR versus bLR Amygdala}

DNMT1 mRNA expression was also compared between bHR/bLR pups within several nuclei of the amygdala, in part because of the role of this region in emotional processing and extensive interconnectivity with the hippocampus. DNMT1 mRNA levels were quantified in 7 amygdalar nuclei (lateral nucleus, central nucleus, basolateral nucleus, medial nucleus, basomedial nucleus, cortical nucleus and the amygdalar-hippocampal transition area), and we discovered significant $b H R / b L R$ differences within 4 of the nuclei, specifically at P7 (fig. 4). At P7, bLR pups exhibited greater DNMT1 mRNA expres- sion in the medial nucleus of the amygdala compared to bHRs $\left(F_{1,18}=6.617, p>0.05\right.$; fig. 2a). Conversely, at P7, bHRs expressed higher DNMT1 levels in the basolateral nucleus $\left(F_{1,25}=15.362, p>0.05\right.$; fig. $\left.2 b\right)$, the lateral nucleus $\left(\mathrm{F}_{1,25}=10.088, \mathrm{p}>0.05\right.$; fig. $\left.2 \mathrm{~d}\right)$; and a trend in the central nucleus $\left(\mathrm{F}_{1,17}=7.771, \mathrm{p}=0.07\right.$; fig. $\left.2 \mathrm{~d}\right)$. There were no bHR/bLR DNMT1 expression differences in these nuclei at P14 and P21, and no differences at any time point within the other amygdalar nuclei that were examined (table 1).

\section{DNMT1 mRNA Expression in Other Brain Areas of $b H R$ versus $b L R$ at $P 7$}

Because we found bHR/bLR DNMT1 mRNA expression differences in both the hippocampus and amygdala at P7, we wanted to examine the specificity of this effect by comparing DNMT1 expression in other brain regions of bHR/bLR pups at this age. Thus, we also assessed DNMT1 mRNA expression in select regions - the cingu- 
Fig. 4. DNMT mRNA expression in bHR versus bLR amygdala at postnatal day 7 . DNMT1 mRNA expression was examined in 7 amygdalar nuclei of bHR and bLR pups at 3 developmental time points: P7, P14, and P21. This analysis found that bHR/bLR differences were restricted to P7 and affected 4 nuclei: the medial nucleus (a), basolateral nucleus (b), the lateral nucleus (c) and central nucleus (d) of the amygdala. At P7, bLRs showed higher DNMT1 mRNA expression compared to bHRs in the medial nucleus (a). However, in the basolateral nucleus, lateral nucleus and central nucleus, bHRs expressed greater DNMT1 at P7. ${ }^{*} \mathrm{p}<0.05$.

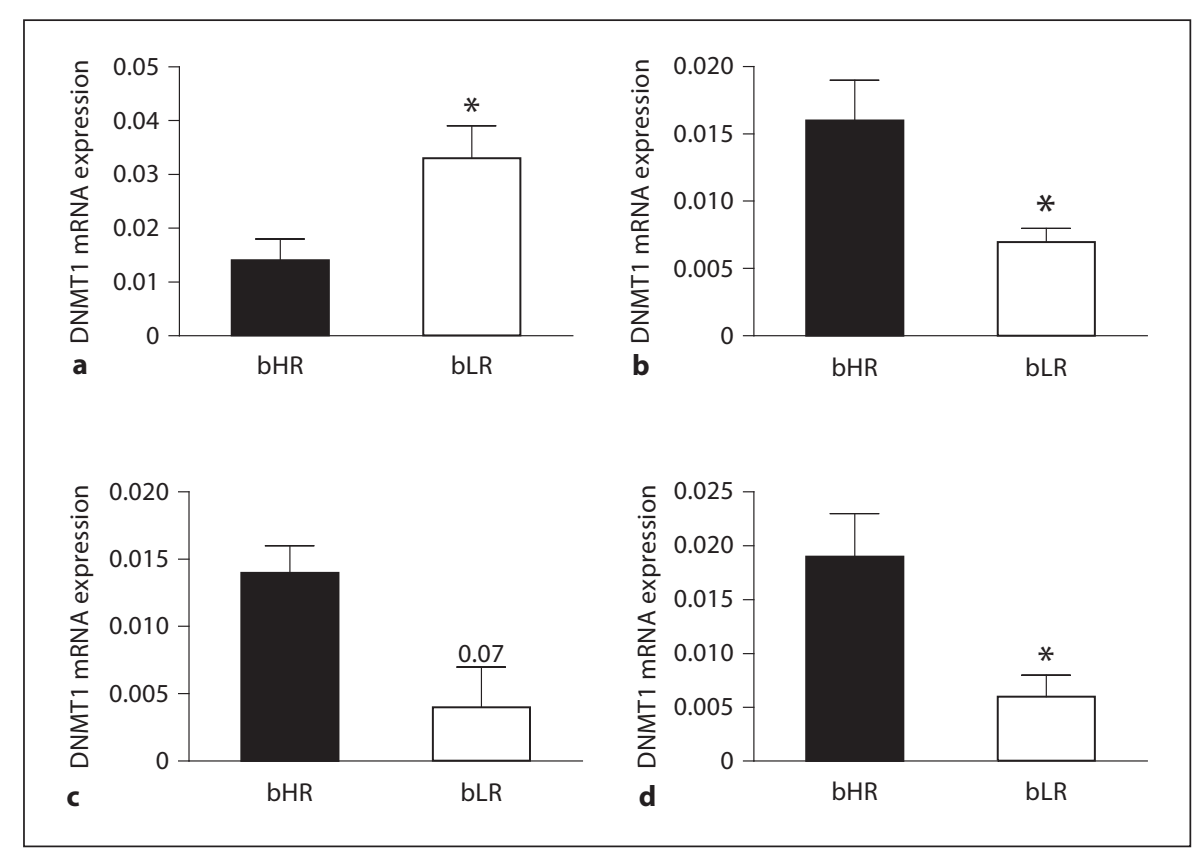

late cortex, caudate putamen, lateral septum and nucleus accumbens, of bHR/bLR P7 rats (fig. 5a). At P7, there were similar DNMT1 mRNA levels in the cingulate cortex (fig. 5b), caudate putamen (fig. 5c), lateral septum (fig. 5d) and nucleus accumbens (fig. 5e) of bHR and bLR pups.

\section{Discussion}

We recently utilized microarray gene expression analysis to demonstrate dramatic gene expression differences in the developing brains of the high-anxiety bLR versus high risk-taking bHR pups. Specifically, we found marked changes in the hippocampus during the first 2 postnatal weeks (P7 and P14), which, together with anatomical analysis of the developing hippocampus in bHR/bLR rats, suggested that differences in hippocampal circuit development may contribute to the divergent bHR/bLR behavioral phenotypes [11]. The present study begins to examine potential epigenetic differences that may drive those bHR/bLR hippocampal gene expression differences, showing that DNMT1 mRNA expression differs in select hippocampal subregions of bHR versus bLR animals at P7. Considering the important role of the amygdala in guiding emotional behavior as well as its significant neural connections with the hippocampus, we also examined DNMT1 expression in several amygdalar nuclei of devel- oping bHR/bLR pups. Here we found significant bHR/ bLR DNMT1 mRNA differences again only at P7 within select nuclei: the medial, basolateral, lateral and central nuclei of the amygdala. Our findings were selective in that we (a) only observed bHR/bLR DNMT1 differences at a single time point (P7 with no changes at P14 and P21), and (b) only found differences in the hippocampus and amygdala, but not other brain areas, including the cingulate cortex, caudate putamen, lateral septum and nucleus accumbens. These findings together with our earlier microrarray work continue to support the notion that the disparate bHR/bLR behavioral phenotypes are at least partially driven by differences in the establishment of hippocampal (and perhaps amygdalar) circuits.

The hippocampus has a long-established role in learning and memory [12]. However, the hippocampus has also been shown to play an important role in behavioral inhibition, including processing emotionally salient information and controlling behavior such as response to novelty, regulating stress, fear and anxiety-like behavior. As noted above, our recent studies revealed marked gene expression differences in the developing bHR/bLR hippocampus [11], and previous work has also reported bHR/bLR hippocampal gene expression differences in adulthood [13-17]. Other studies have shown clear differences in the structure of bHR/bLR hippocampus in adult rats, with adult bLRs having increased cell proliferation and neuron number in the dentate gyrus as well as increased hippo- 
Fig. 5. DNMT1 mRNA expression in multiple forebrain regions of bHR versus bLR pups at P7. We compared DNMT1 levels in $\mathrm{P} 7 \mathrm{bHR} / \mathrm{bLR}$ rats in the cingulate cortex $(\mathrm{Cg})$, caudate putamen $(\mathrm{CPu})$, lateral septum (LS) and nucleus accumbens (NAc) to determine whether bHR/bLR DMNT1 differences at this age were widespread, or specific to the hippocampus and amygdala. a Shows an autoradiogram of a representative section through these brain regions to illustrate the pattern of DNMT1 mRNA expression in these four areas. Quantification of these data showed that there were no bHR/bLR differences in DNMT1 mRNA levels in the cingulate (b), caudate putamen (c), septum (d) and nucleus accumbens (e).

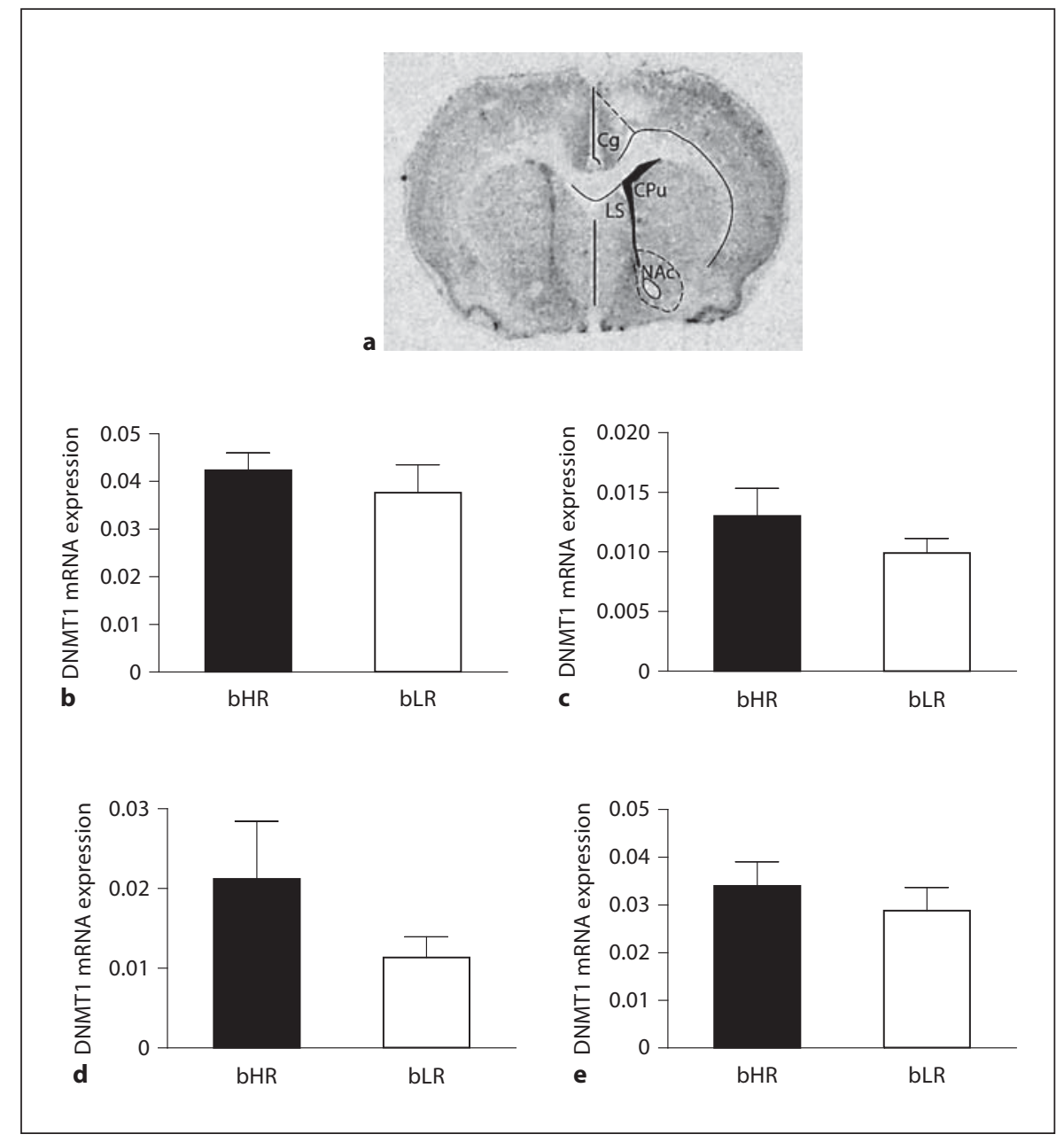

campal volume and a larger volume of superpyramidal mossy fibers $[18,19]$. Moreover, we observed increased hippocampal volume and enhanced cell proliferation in the developing bLR versus bHR hippocampus [11]. Together these data suggest that a potential augmentation of hippocampal connectivity and/or functioning in bLR versus bHR rats may contribute to an exaggerated degree of behavioral inhibition $[18,20]$. We therefore suggest that essential features of bHR/bLR structure and function of the hippocampus and related circuitry drive their differences in environmental reactivity.

Our present DNMT1 mRNA data suggest that bHR/ bLR hippocampal differences may be accompanied (or perhaps driven) by underlying epigenetic differences, although future work will be required to more fully examine putative bHR/bLR methylation differences and downstream effects. Prior evidence suggests that mRNA levels of DNMTs and other methylation-related molecules of- ten correlate with global methylation levels in tissue [1, $21,22]$, so one interpretation of our DNMT1 findings is that bLRs exhibit increased methylation within select hippocampal subregions at P7 compared to bHRs. Because the present study only examines one of the three known DNMTs (DNMT1), future work should also include an examination of DNMT3a and DNMT3b as well as other methylation-related molecules in bHR/bLR hippocampus, and should extend to examine global methylation in the hippocampus and other brain regions of developing bHR/bLR rats. Moreover, in addition to characterizing global methylation patterns, future efforts will examine what specific targets may be differentially methylated in bHR/bLR rats, and whether pharmacological manipulation of methylation status significantly impacts gene expression and behavioral differences that are commonly observed in bHR/bLR rats. Considering the complexity of methylation processes and how they impact 
downstream gene expression and cell function, we need to carefully step through and analyze different facets of epigenetic regulation in bHR/bLR animals (from early life through adulthood) to fully understand how they differ and how epigenetic differences may contribute to their divergent behavior.

It was interesting that we found increased DNMT1 mRNA expression in P7 bLRs versus bHRs specifically in the upper blade of the dentate gyrus, with no differences occurring in the lower blade of the dentate. In 2005, Chawla et al. [23] found differential expression of behaviorally induced immediate early gene Arc in the upper blade of the dentate versus the lower blade of the dentate in normal adult rats. They showed that there was no behaviorally dependent Arc activation in the lower dentate gyrus compared to the upper dentate gyrus, which suggests that the dentate gyrus responds specifically to different environmental and/or special cues. Our data provide additional support to the idea of a possible differential function in the upper versus lower blades of the dentate gyrus although future work will be required to more fully investigate this issue.

Just as the hippocampus plays a critical role in the regulation of emotional behavior and stress reactivity, the amygdala also plays a crucial role in these processes, functioning as an interface between incoming emotionally salient sensory information from the environment, and subsequent behavioral responses. Thus, the hippocampus and amygdala cooperate to control anxiety-like behavior, and a perturbation of hippocampal-amygdala circuits likely contributes to emotional dysregulation in anxiety and mood disorders in humans. Dysfunction within either the hippocampus or amygdala, or altered connectivity between these regions, could engender an excessive perception of threat and thus give rise to an overly anxious state $[24,25]$.

The present study revealed bHR/bLR DNMT1 mRNA differences at P7 within select nuclei of the amygdala: the medial, basolateral, lateral, and central nuclei. This is not the first observation of amygdalar differences in HR/LR rats, as one previous study found increased expression of corticotropin-releasing hormone $(\mathrm{CRH})$ in the amygdala of adult LR versus HR rats, which is consistent with their exaggerated anxiety-like behavior [14]. It would be interesting to see whether such differences exist in the developing bLR versus bHR brain, and whether such differences may be linked to underlying bHR/bLR differences in methylation. With regard to the functional significance of the specific nuclei where we observed bHR/bLR DNMT1 expression differences, it is certainly interesting that the basolateral amygdala was one nucleus where bHR/bLR differences were apparent. The basolateral amygdala is known to play a significant role in modulating emotionally charged memory formation, and both the basolateral and lateral nuclei reciprocally connect with the hippocampus to process emotionally salient sensory information and guide anxiety-like behavioral response $[24,25]$. The central nucleus of the amygdala plays a different, but certainly important role in fear conditioning by coordinating fear-related behavioral and physiological responses, including freezing behavior, increased respiration and stress hormone release [26-28]. Considering the abundant evidence for bHR/bLR differences in anxiety-like behavior $[8,9,11,14,29-31]$, it would not be at all surprising to also find differences in fear and emotional learning in bHR and bLR rats. Future efforts will test this notion, and also explore possible neural correlates of these differences in the amygdala and other brain regions of these animals. Because emotional learning involves multiple brain areas working together, future work will expand to look at methylation patterns in other limbic regions, such as the prefrontal cortex, that likely functionally differ between bHR/bLR rats.

In our previous work, we considered not only genetic and innate biological differences that may contribute to the disparate bHR/bLR behavioral phenotypes, but have also explored how early life experience influences the bHR versus bLR brain and subsequent behavior $[8,9]$. Elegant work by Meaney [32] has shown how maternal behavior critically shapes brain development and emotional behavior. He showed how highly maternal rat mothers raise offspring with diminished stress reactivity and anxiety compared to offspring of low licking, grooming and arched-back nursing mothers, and that these effects were driven, in part, through changes in the hippocampus, including epigenetic modifications in the promoter region of the glucocorticoid receptor [32]. Based on his findings, we wondered whether bHR versus bLR dams acted differently with their pups and whether such differences may drive the distinct bHR versus bLR behavioral phenotypes. We found that bLR/ bHR mothers do in fact exhibit distinct maternal styles, with bLR mothers generally acting more maternally (showing more licking, grooming, arched-back nursing) of pups compared to bHR mothers. Although bHR dams exhibited less maternal behavior than bLRs during the dark/active phase, they were very attentive to their pups during the light phase, spending more time passive nursing and in contact with pups compared to bLR dams $[33,34]$. 
We also conducted a cross-foster study to test whether bHR/bLR maternal differences impacted the behavior of offspring. Cross-fostering had absolutely no impact on locomotor response to novelty (all bHR groups showed very high levels of novelty-induced activity compared to the bLR groups). Interestingly, though, cross-fostering subtly improved the anxiety of bLRs, with bLRs raised by biological bLR mothers showing high anxiety relative to bLRs raised by either a bHR or a bLR foster mother [8]. Curiously, these results suggest that it was not the bHR or bLR maternal style per se that positively impacted the anxiety of bLRs; rather, the fact that they were fostered to another dam (whether bHR or bLR) positively influenced their ultimate level of anxiety behavior. Overall these results suggest that while bHR/bLR mothers exhibit differences in how they interact with their young, most aspects of their offspring's behavioral phenotypes derive from underlying genetic differences. It remains to be seen what effect cross-fostering may have on neural differences observed in bHR/bLR rats - such as widespread gene expression differences in the hippocampus, or possible differences in DNA methylation. It would be extremely interesting to see whether the protective/anxiolytic effect of foster care on the adult behavior of bLRs corresponds with gene expression and methylation differences in the hippocampus. The putative P7 methylation differences in hippocampus and amygdala (indicated by our present data) may well be related to the basic biological signature of bHR versus bLR developmental neurobiology. Alternatively, we cannot exclude the possibility that those differences are related to known bHR/bLR maternal care differences and only further experiments will definitively answer this question. Future studies will combine crossfostering with examination of gene expression and methylation differences in limbic brain regions of bHR/bLR rats to investigate this possibility.

Epigenetic modifications play an important role during development, and throughout life. Previous research has shown many important roles for DNMT during embryogenesis and adulthood; however, limited research has focused on the role of DNMTs in postnatal development. During embryonic development, the epigenome goes through stages of methylation and demethylation in order to achieve normal development. As a result, DNA methylation must occur at very specific times during development and is very well regulated. During germ cell development and early embryogenesis, global de novo methylation takes place. How DNMTs play a role in this global de novo methylation is still unclear, although several possibilities have been reported [4]. Studies have also shown region-specific variations in DNMT expression in adult hippocampus. These studies and others have proposed that certain DNA methylation patterns undergo constant remodeling in response to environmental cues throughout adulthood and that DNMT plays an important role in permitting that adaptation [21]. Our data support the concept of DNA methylation playing an important role throughout life, specifically in a period of early postnatal development.

\section{Acknowledgements}

We are very grateful to Sue Miller for excellent technical assistance, and grateful to the laboratory of Dr. Stanley Watson and Sharon Burke, who developed the DNMT1 riboprobe and provided it to us for experiments. The study was funded by NIMH 4R00MH085859-02 (S.M.C.), Office of Naval Research ONRN00014-09-1-0598 (H.A.), and NIDA PPG 5P01DA021633-02 (H.A.).

\section{Disclosure Statement}

There are no biomedical financial interests or conflicts of interest for any of the authors.

\section{References}

1 Sweatt JD, Miller CA: Covalent modification of DNA regulates memory formation. Neuron 2007;53:857-869.

2 Lubin FD, Roth TL, Sweatt JD: Epigenetic regulation of $\mathrm{BDNF}$ gene transcription in the consolidation of fear memory. J Neurosci 2008;28:10576-10586.

3 Chen ZX, Riggs AD: DNA methylation and demethylation in mammals. J Biol Chem 2011;286:18347-18353.
4 Feng J, Fan G: The role of DNA methylation in the central nervous system and neuropsychiatric disorders. Int Rev Neurobiol 2009; 89:67-84.

5 Flagel SB, Robinson TE, Clark JJ, Clinton SM, Watson SJ, Seeman P, Phillips PE, Akil $\mathrm{H}$ : An animal model of genetic vulnerability to behavioral disinhibition and responsiveness to reward-related cues: implications for addiction. Neuropsychopharmacology 2010; 35:388-400.
6 Clinton SM, Kerman IA, Orr HR, Bedrosian TA, Abraham AD, Simpson DN, Watson SJ, Akil H: Pattern of forebrain activation in high novelty-seeking rats following aggressive encounter. Brain Res 2011;1422:20-31.

7 Kerman IA, Clinton SM, Bedrosian TA, Abraham AD, Rosenthal DT, Akil H, Watson SJ: High novelty-seeking predicts aggression and gene expression differences within defined serotonergic cell groups. Brain Res 2011;1419:34-45. 
\$ Stead JD, Clinton S, Neal C, Schneider J, Jama A, Miller S, Vazquez DM, Watson SJ, Akil H: Selective breeding for divergence in novelty-seeking traits: heritability and enrichment in spontaneous anxiety-related behaviors. Behav Genet 2006;36:697-712.

-9 Clinton S, Miller S, Watson SJ, Akil H: Prenatal stress does not alter innate noveltyseeking behavioral traits, but differentially affects individual differences in neuroendocrine stress responsivity. Psychoneuroendocrinology 2008;33:162-177.

10 Stedenfeld KA, Clinton SM, Kerman IA, Akil H, Watson SJ, Sved AF: Novelty-seeking behavior predicts vulnerability in a rodent model of depression. Physiol Behav 2011;103: 210-216.

-11 Clinton SM, Stead JD, Miller S, Watson SJ, Akil H: Developmental underpinnings of differences in rodent novelty-seeking and emotional reactivity. Eur J Neurosci 2011;34: 994-1005.

12 Eichenbaum H, Otto T, Cohen NJ: The hippocampus - what does it do? Behav Neural Biol 1992;57:2-36.

13 Rosario LA, Abercrombie ED: Individual differences in behavioral reactivity: correlation with stress-induced norepinephrine efflux in the hippocampus of Sprague-Dawley rats. Brain Res Bull 1999;48:595-602.

14 Kabbaj M, Devine DP, Savage VR, Akil H: Neurobiological correlates of individual differences in novelty-seeking behavior in the rat: Differential expression of stress-related molecules. J Neurosci 2000;20:6983-6988.

15 Kabbaj M: Neurobiological bases of individual differences in emotional and stress responsiveness: High responders-low responders model. Arch Neurol 2004;61:1009-1012.

16 Kabbaj M, Evans S, Watson SJ, Akil H: The search for the neurobiological basis of vulnerability to drug abuse: using microarrays to investigate the role of stress and individual differences. Neuropharmacology 2004; 47(suppl 1):111-122.
Ballaz SJ, Akil H, Watson SJ: Analysis of 5-HT6 and 5-HT7 receptor gene expression in rats showing differences in novelty-seeking behavior. Neuroscience 2007;147:428438.

18 Lemaire V, Aurousseau C, Le Moal M, Abrous DN: Behavioural trait of reactivity to novelty is related to hippocampal neurogenesis. Eur J Neurosci 1999;11:4006-4014.

19 Isgor C, Slomianka L, Watson SJ: Hippocampal mossy fibre terminal field size is differentially affected in a rat model of risk-taking behaviour. Behav Brain Res 2004;153:7-14.

20 McNaughton N: Cognitive dysfunction resulting from hippocampal hyperactivity - a possible cause of anxiety disorder? Pharmacol Biochem Behav 1997;56:603-611.

1 Brown SE, Weaver IC, Meaney MJ, Szyf M: Regional-specific global cytosine methylation and DNA methyltransferase expression in the adult rat hippocampus. Neurosci Lett 2008;440:49-53.

22 Miller CA, Campbell SL, Sweatt JD: DNA methylation and histone acetylation work in concert to regulate memory formation and synaptic plasticity. Neurobiol Learn Mem 2008;89:599-603.

23 Chawla MK, Guzowski JF, Ramirez-Amaya V, Lipa P, Hoffman KL, Marriott LK, Worley PF, McNaughton BL, Barnes CA: Sparse, environmentally selective expression of Arc RNA in the upper blade of the rodent fascia dentata by brief spatial experience. Hippocampus 2005;15:579-586.

-24 Da Cunha C, Roozendaal B, Vazdarjanova A, McGaugh JL: Microinfusions of flumazenil into the basolateral but not the central nucleus of the amygdala enhance memory consolidation in rats. Neurobiol Learn Mem 1999;72:1-7.

25 Vazdarjanova A, McGaugh JL: Basolateral amygdala is involved in modulating consolidation of memory for classical fear conditioning. J Neurosci 1999;19:6615-6622.

26 Campeau S, Davis M: Involvement of the central nucleus and basolateral complex of the amygdala in fear conditioning measured with fear-potentiated startle in rats trained concurrently with auditory and visual conditioned stimuli. J Neurosci 1995;15:23012311.
27 Phillips RG, LeDoux JE: Differential contribution of amygdala and hippocampus to cued and contextual fear conditioning. Behav Neurosci 1992;106:274-285.

-28 Wilensky AE, Schafe GE, Kristensen MP, LeDoux JE: Rethinking the fear circuit: The central nucleus of the amygdala is required for the acquisition, consolidation, and expression of pavlovian fear conditioning. J Neurosci 2006;26:12387-12396.

29 White DA, Kalinichev M, Holtzman SG: Locomotor response to novelty as a predictor of reactivity to aversive stimuli in the rat. Brain Res 2007;1149:141-148.

30 Perez JA, Clinton SM, Turner CA, Watson SJ, Akil H: A new role for FGF2 as an endogenous inhibitor of anxiety. J Neurosci 2009; 29:6379-6387.

- 31 Turner CA, Clinton SM, Thompson RC, Watson SJ Jr, Akil H: Fibroblast growth factor-2 (FGF2) augmentation early in life alters hippocampal development and rescues the anxiety phenotype in vulnerable animals. Proc Natl Acad Sci USA 2011;108:8021-8025.

32 Meaney MJ: Maternal care, gene expression, and the transmission of individual differences in stress reactivity across generations. Annu Rev Neurosci 2001;24:1161-1192.

33 Clinton SM, Vazquez DM, Kabbaj M, Kabbaj $\mathrm{MH}$, Watson SJ, Akil H: Individual differences in novelty-seeking and emotional reactivity correlate with variation in maternal behavior. Horm Behav 2007;51:655-664.

- 34 Clinton SM, Bedrosian TA, Abraham AD, Watson SJ, Akil H: Neural and environmental factors impacting maternal behavior differences in high- versus low-novelty-seeking rats. Horm Behav 2010;57:463-473.

35 Paxinos G, Watson C: The Rat Brain in Stereotaxic Coordinates, ed 3. San Diego, Academic Press, 1997. 